Center for Neurosciences, The Feinstein Institute for Medical Research, 350 Community Drive, Manhasset, NY 11030. E-mail: afeigin@nshs.edu).

COMMENT. Metabolic brain network patterns differentiate subjects with TS from controls, as well as a second pattern that differentiates TS subjects with OCD from those without OCD. The brain networks involve regions associated with motor activity as well as those regions associated with behavioral changes (anterior cingulate and prefrontal cortex).

In an editorial, Dr Katie Kompoliti of Rush Med Sch, Chicago comments that this study identifies TS-related abnormal network patterns that encompass multiple interacting nuclei instead of isolated regions, a view of the whole "elephant," not just the trunk or tail. (Neurology March 15, 2011;76:938-939). She remarks that the study is limited by differences in gender mix of subjects (mainly male) and controls (mainly female). Other limitations include the age of subjects (all adults whose tics are usually severe), and the absence of additional TS comorbidities such as ADHD that might influence results.

\title{
AZITHROMYCIN-ASSOCIATED CHOREOATHETOSIS
}

Researchers at Women and Children's Hospital of Buffalo, NY, report an 11year-old boy with a history of developmental delay who developed transient agitation and choreoathetoid movements of upper extremities in temporal relation to treatment of influenza A and B respiratory infections with oral azithromycin on 2 occasions. Symptoms improved with brief administration of clonazepam or lorazepam, and they resolved within 36-48 hours of discontinuation of azithromycin. The association of agitation and movement disorder with azithromycin is previously unreported. A causal relation was considered probable, based on a score of 6 on the Naranjo adverse drug reaction probability scale. (Farooq O, Memon Z, Stojanovski SD, Faden HS. Azithromycin-induced agitation and choreoathetosis. Pediatr Neurol 2011;44:311-313). (Respond: Dr Farooq, Women and Children's Hospital, Division of Pediatric Neurology, 219 Bryant Street, Buffalo, NY 14222. E-mail: osmanfarooq@yahoo.com).

COMMENT. Macrolide antibiotics, especially clarithromycin, are known to cause acute psychoses when given with amoxicillin. A syndrome known as "Hoigne syndrome" or "antibiomania" consisting of delusions, paranoia, and hallucinations is reported. One patient developed catatonia during azithromycin treatment. In the present case-report of choreoathetosis with azithromycin, the association of influenza viral infection is a possible factor in etiology.

\section{NEUROPATHIES}

\section{PEDIATRIC SCIATIC NEUROPATHIES}

Prospective review of the incidence, cause, and prognosis of pediatric sciatic neuropathy $(\mathrm{SN})$ in a 30 -year experience of 53 patients is reported from the Department 
of Neurology, Children's Hospital Boston, Lahey Clinic, Burlington, MA, and Royal Children's Hospital, Melbourne, Australia. Ages ranged from 2 days to 18 years. Mononeuropathies in children are rare, constituting $<10 \%$ of referrals for EMG. Most patients presented with tibialis anterior weakness and foot drop. Weakness of plantar flexion was seen in $31(58 \%)$ patients. Numbness below the knee occurred in all patients able to describe the symptom. The causes of SN included trauma (13), iatrogenic mechanisms related to orthopedic surgeries (13), extrinisic compression and immobilization (6), tumors (7), vascular (5), idiopathic and progressive (4), infantile and nonprogressive (2), and presumed postviral (3). Motor conduction was abnormal in 44/53 (83\%) peroneal nerve studies, and 35/51 (67\%) tibial nerve. Sensory conduction studies were abnormal in sural nerve in 34 of 43 cases (70\%), and in superficial peroneal nerves in $15 / 25(60 \%)$. Needle EMG was abnormal in peroneal innervated muscles in all subjects, in tibial nerve innervated muscles in $43 / 51(84 \%)$, and in the hamstrings in $18 / 29(62 \%)$. Prognosis was variable and dependent on etiology and severity of nerve injury; good in traumatic and iatrogenic SN and poor in vascular or tumor cases. (Srinivasan J, Ryan MM, Escolar DM, Darras B, Jones HR. Pediatric sciatic neuropathies: a 30-year prospective study. Neurology March 15, 2011;76:976-980). (Response and reprints: Dr Jayashri Srinivasan, Department of Neurology, Lahey Clinic, 41 Mall Road, Burlington, MA 01805. E-mail: jayashri_srinivasan@lahey.org).

COMMENT. Electrophyiological studies are important in diagnosis of neuropathies in children but are limited by poor tolerance. MRI will become increasingly useful with higher resolution units. Causes of SN are more varied in children than in adults. Traumatic causes predominate.

\section{HEADACHE DISORDERS}

\section{ROLE OF NEUROIMAGING IN RECURRENT HEADACHES}

Researchers at multiple centers in Korea have retrospectively reviewed the records of all 1562 (male 724, female 838) new patients presenting with recurrent headaches in 9 Pediatric Neurology Clinics of tertiary hospitals. Headaches were classified according to the International Classification, $2^{\text {nd }}$ ed. Neuroimaging was performed in $77.1 \%$ of patients, and overall, 9.3\% (112/1204) were abnormal; $2.3 \%$ $(5 / 214)$ in CTs, $8.6 \%(88 / 1022)$ in MRI, and $17.6 \%$ (19/108) in MRI and MR angiograms. The mean age of onset of headache was 8 years, the mean frequency of attacks was 13/month, and the mean duration of symptoms at presentation was 16 months. The highest yield was obtained in patients with an abnormal neurological examination, and 50\% $(9 / 18)$ were abnormal $(\mathrm{P}<.001)$. Lowest yields occurred when imaging was performed for changes in type of headache (12\% [26/201]), neurologic dysfunction (10\% [9/83]). recent onset of severe headaches (7\% [12/171]), and demands of parent and physician (10\% [21/208]). Surgery was performed in $0.9 \%$ patients who underwent neuroimaging, and in $9.8 \%$ with abnormal findings. Neuroimaging showed no significant relation with age, sex, headache type, age of onset, duration of symptoms, frequency, location, or intensity of headache $(\mathrm{P}>.05)$. The findings suggest that stricter guidelines are needed for neuroimaging in pediatric headache patients. (Rho Y-II, Chung 